\title{
CD33 and TREM2 peripheral gene expression in relation to cortical thickness
}

\author{
Abby L. Braun, BS ${ }^{1}$, Apoorva Bharthur Sanjay, $\mathrm{MS}^{2}$, Diana O. Svaldi, Liana G. \\ Apostolova, $\mathrm{MD}^{2,3}$ \\ ${ }^{1}$ Indiana University School of Medicine, ${ }^{2}$ Indiana University School of Medicine, \\ Department of Neurology, ${ }^{3}$ Indiana University School of Medicine, Indiana \\ Alzheimer Disease Center
}

Background and Hypothesis: Genome-wide association and whole genome sequencing studies have uncovered over 20 novel Alzheimer's Disease (AD) risk genes. Many of these genes are involved with neuroinflammation.

Neuroinflammation plays a pivotal role in disease development. CD33 and TREM2 are two immune pathway genes implicated in AD. We studied the association between peripheral blood expression of CD33 and TREM2, with cortical thickness - a marker of neurodegeneration in AD. We hypothesized that expression of some these genes would correlate with cortical atrophy in our pooled sample.

Experimental Design or Project Methods: Data from 155 subjects (50 NC and $105 \mathrm{MCl}$ ) were gathered from the UCLA ImaGene study. The imaging data was processed using the FreeSurfer 6.0 longitudinal pipeline and cortical anatomy was standardized for all subjects. A GLM analysis using Surfstat investigated the associstion of gene expression levels in peripheral blood and cortical thickness controlling for age, gender, education, and diagnosis and generated $3 \mathrm{D}$ visualization maps of these associations. Statistical significance was determined with $p$ set at 0.05 .

Results: $C D 33$ expression showed a positive correlation with cortical thickness in the right medial, inferior and lateral temporal, lateral parietal, posterior cingulate and inferior lateral frontal cortices. TREM2 did not show statistically significant association with cortical atrophy.

Conclusion and Potential Impact: Peripeheral blood CD33 expression level seems to be lower in individuals with greater cortical atrophy in AD-relevant areas. This contributes to a growing body of research that indicates a role for the immune system and $C D 33$ in particular in the pathology of $A D$ and may contribute to future mechanism and biomarker studies. 